\title{
ESTUDO COMPARATIVO SOBRE O USO DE MÍDIA SOCIAL EM ESCOLAS
}

\author{
Elias Estevão GOULART ${ }^{1}$ \\ Päivi AARRENIEMI-JOKIPELTO ${ }^{2}$
}

Resumo: A presença do Facebook, um canal de comunicação quase universal, está indubitavelmente transformando as escolas. Um acompanhamento de 12 escolas finlandesas e brasileiras foi realizado para entender como o Facebook tem sido empregado. A teoria da presença social foi selecionada como referencial teórico das análises. A similaridade mais importante encontrada foi a presença coesiva em ambas as comunidades. A despeito dos dados serem oriundos dos perfis públicos das instituições, certamente outros usos específicos da mídia social pelas escolas podem ser explorados.

Palavras-chave: Mídia social. Facebook. Presença social. Ação pedagógica.

\section{Introdução}

Nossa sociedade alcançou um nível especial de interconexão entre as pessoas, por meio de enorme quantidade de enlaces multiformes on-line (ou virtual), que enriqueceu todos os seus aspectos, primeiro no contexto familiar, então na escola, e na participação comunitária onde se trabalha e vive. Nossos relacionamentos oferecem o os fundamentos e suporte para a melhoria das esferas sociais. "As pessoas encontram-se, cada vez mais, interconectando-se com o auxílio da Internet, não importando cor, riqueza, religião ou profissão, criando vínculos e laços que, muito provavelmente, não existiriam sem a rede." (GOULART; RAMOS; CRUZ, 2012).

Os relacionamentos são criados pelos indivíduos dentro de um grupo e geram oportunidades para as trocas culturais sobre enorme diversidade de atividades, como discussões de assuntos de interesse mútuo, contribuindo para projetos de importância para eles, em aprendizagens etc. As tecnologias empoderam o ser humano e as mídias sociais podem criar inúmeras novas formas de integração das pessoas pela amplificação de suas conexões e da maneira como comunicam. Relacionamentos pessoais podem ser

${ }^{1}$ USCS - Universidade Municipal de São Caetano do Sul. São Caetano do Sul - SP - Brasil. 09150-050. Centro Universitário Fundação Santo André. Santo André - SP - Brasil. 09060-650 elias.goulart@uscs.edu.br

${ }^{2}$ HAAGA-HELIA - University of Applied Sciences. Helsinki Area - Finlândia. 00700 - paivi.aarreniemijokipelto@haaga-helia.fi 
estabelecidos com o uso das mídias sociais e das comunidades virtuais. Mais do que as simples transmissões de mensagens entre os integrantes dos grupos, sua coesão é percebida e construída de acordo com seus interesses e motivações comuns, de uma forma não sistemática, dentro da dinâmica das comunidades.

A mídia social pode ser definida como um conjunto de atividades práticas e comportamentos entre pessoas e comunidades de pessoas que se encontram on-line por meio de websites que subsidiam a interação social, a criação colaborativa de conteúdos e o compartilhamento de informações, conhecimento e opiniões em vários formatos - usando mídias virtuais gratuitas e abertas para colaboração, conversação e interação de todos, sem intermediação direta, e na qual o 'usuário' torna-se produtor e consumidor de informação. Um dos desafios atuais tem sido caracterizar as mídias sociais para compreender sua operação e o que faz as pessoas avidamente desejarem se conectar virtualmente, como apontam Cavazza (2014), Goulart (2014), Kietzmann (2012), dentre outros. A Tabela 1 mostra as características básicas das mídias sociais que ajudam analisar sua forma de trabalho, bem como as implicações de seu uso. Uma preocupação fundamental é a percepção do outro: como alguém pode estar certo sobre a outra pessoa ou como a tecnologia pode mediar as interações ajudando-os a se encontrarem virtualmente?

As medidas indicadas na Tabela 1 permitem analisar a efetividade das mídias sociais e sua habilidade para atrair grande número de usuários. Especificamente, a presença é a percepção do outro por meio de uma conexão virtual cuja resistência e duração dependerão do contexto envolvido, da habilidade para criar intimidade e todos os elementos para a construção da confiança. 
Tabela 1 - Características fundamentais das mídias sociais

\begin{tabular}{|c|c|c|}
\hline CARACTERISTICA & FUNCIONALIDADE & IMPLICAÇÕES \\
\hline Identidade & $\begin{array}{l}\text { Medida da auto revelação dos } \\
\text { usuários. }\end{array}$ & $\begin{array}{l}\text { Controle da privacidade dos } \\
\text { dados e ferramentas para os } \\
\text { usuários se auto promover. }\end{array}$ \\
\hline Conversação & $\begin{array}{l}\text { Medida sobre a comunicação inter } \\
\text { usuários. }\end{array}$ & $\begin{array}{l}\text { Velocidade das conversas e } \\
\text { riscos de começar e se juntar } \\
\text { a conversas. }\end{array}$ \\
\hline Compartilhamento & $\begin{array}{l}\text { Medida sobre a troca, distribuição e } \\
\text { recepção de conteúdos. }\end{array}$ & $\begin{array}{l}\text { Sistema para gestão de } \\
\text { conteúdos e interconexões } \\
\text { sociais. }\end{array}$ \\
\hline Presença & $\begin{array}{l}\text { Medida da disponibilidade dos } \\
\text { demais usuários. }\end{array}$ & $\begin{array}{l}\text { Criação e gestão da realidade, } \\
\text { intimidade e urgência do } \\
\text { contexto. }\end{array}$ \\
\hline Relacionamento & $\begin{array}{l}\text { Medida sobre as relações entre } \\
\text { usuários. }\end{array}$ & $\begin{array}{l}\text { Gestão das propriedades } \\
\text { estruturais e fluxos na rede de } \\
\text { relacionamentos. }\end{array}$ \\
\hline Reputação & $\begin{array}{l}\text { Medida do conhecimento sobre a } \\
\text { posição social dos outros. }\end{array}$ & $\begin{array}{l}\text { Monitoramento do poder, } \\
\text { paixão, sentimentos e } \\
\text { abrangência dos usuários e } \\
\text { marcas. }\end{array}$ \\
\hline Agrupamento & $\begin{array}{l}\text { Medida sobre os usuários se estão } \\
\text { prontos ou formam comunidades. }\end{array}$ & $\begin{array}{l}\text { Regras e protocolos da } \\
\text { membresia. }\end{array}$ \\
\hline
\end{tabular}

Fonte: Ribeiro Neto (2015, p.26).

A mídia social chamada Facebook é a conexão virtual de maior utilização para o encontro, compartilhamento, interação e discussão de ideias. Fundado em fevereiro de 2004 por Mark Zuckerberg, um estudante da Universidade de Harvard, foi originalmente projetado para integrar os estudantes universitários em uma plataforma de fácil acesso e interações. O Facebook é adequado para o compartilhamento de mídias, interesses comuns e seu foco primário está na comunicação assíncrona (SCHÖN; EBNER, 2015). A penetração do Facebook no Brasil foi de $83.8 \%$ em fevereiro de 2015 e na Finlândia foi de $47.5 \%$ em novembro de 2015 (INTERNET WORLD STATISTICS, 2015). Contudo, a penetração da Internet no Brasil foi de $53.3 \%$ e de 93.5\% na Finlândia (INTERNET WORLD STATISTICS, 2015). Sua presença e domínio no espaço virtual nos permite inferior em que extensão ele influencia e altera a nossa sociedade. Como a pesquisadora Silvia Rosenthal (2015) postula: "The world we live in is not changing back." 
Informação atual sobre o uso e adoção das mídias sociais mostra os jovens com alta utilização, chegando a 75\% dos 'milenares' (de 18 a 29 anos de idade) com perfil on-line (MILLENNIALS..., 2010). Esse quadro levanta as seguintes questões: As escolas têm se apropriado dessa mídia social para trocar conteúdos educacionais ou culturais? De que formas o Facebook tem sido empregado? As escolas têm aplicado ou feito sugestões de aplicação do Facebook nos processos de ensino e aprendizagem? Em um mundo com transformações velozes e radicais, as mídias sociais podem representar uma tecnologia poderosa para melhorar a construção de conhecimento. "We need to ensure that students are equipped with the skills to safely and smartly sift through this abundance of information and to navigate online spaces in ways that contribute to their learning." (COUROS; HILDEBRANDT, 2015).

O principal objetivo desse artigo é investigar como escolas nas cidades de São Caetano do Sul (Brasil) e Helsinki (Finlândia) estão usando o Facebook para se comunicar com suas respectivas comunidades. Os dados obtidos nesses dois países tão diferentes foram comparados, segundo o conceito de presença social.

\section{Presença social}

A presença social, uma das categorias de presença com - por sua própria natureza - amplos, complexos e multidisciplinares aspectos, tem sido largamente discutida e sem consenso entre os pesquisadores, como exemplos, Baren e Ijsselsteijn (2004), Lombard e Jones (2007) etc. A experiência da presença social é, por definição, mediada e seu conceito opera de forma a reduzir a diferença entre ambas as experiências: direta (face-a-face) e mediada. A teoria da presença social envolve a midiatização, enquanto processo, ou seja, postula que a qualidade da comunicação entre duas ou mais pessoas depende do meio que as interliga, conforme a citação: “[...] focuses on the effects of mediation on experience especially as our awareness of the mediation oscillates, flickers, and sometimes fades [...]" (BIOCCA; BURGOON et al., 2001). A meta da presença implícita é ultrapassar a experiência mediada do 'estar lá' para a percepção ou sensação de simplesmente 'estar' dentro de situações ou ambientes mediados. O autor Rourke colegas apresentam uma taxonomia de presença social segundo três dimensões, conforme a tabela 2 abaixo (ROURKE, ANDERSON et al., 2001). 
Tabela 2 - Categorias de presença social

\begin{tabular}{|l|l|}
\hline Categoria & \multicolumn{1}{c|}{ Definição e Indicadores } \\
\hline Afetiva & $\begin{array}{l}\text { - Expressão das emoç̃es e sentimentos: expressões convencionais de emoção } \\
\text { incluem pontuação repetida, uso enfático de caixa alta, 'emoticons'. } \\
\text { - Uso de Humor: provocação, bajulação, ironia, eufemismo, sarcasmo. } \\
\text { - Auto revelação do participante: detalhes pessoais, ambiente profissional ou } \\
\text { vulnerabilidades. }\end{array}$ \\
\hline Interativa & $\begin{array}{l}\text { - Continuação de um tópico: uso da aplicação para "comentar" um tópico, ao } \\
\text { invés de iniciar um novo tópico. } \\
\text { - Citação de mensagens de outros: uso da aplicação para nomear partes ou } \\
\text { mensagem inteira de outras pessoas. } \\
\text { - Menção direta e explicita do conteúdo de postagem de terceiros. } \\
\text { - Fazer perguntas: o usuário faz perguntas ao moderador. } \\
\text { - Cumprimentos, expressões de apreciação, concordância sobre postagens de } \\
\text { outros. }\end{array}$ \\
\hline Coesiva & $\begin{array}{l}\text { - Uso de vocativos: nomear ou se referir ao nome de outro participante. } \\
\text { - Uso de pronomes inclusivos para se referir ao grupo, como 'nós', 'nosso'. } \\
\text { - Uso de expressões factuais, agradecimentos: função puramente social da } \\
\text { comunicação, agradecimentos, despedidas. }\end{array}$ \\
\hline
\end{tabular}

Fonte: Rourke et al. (2001).

Atualmente, o conceito de presença social possui grande importância prática na criação e avaliação de novas mídias e interfaces homem-máquina, especialmente para entretenimento (filmes, TV, shows, videogames), nas telecomunicações (trabalho colaborativo, videoconferência), educação (e-learning, simulações) e cuidados de saúde (telemedicina, telecirurgia), dentre outros usos (LEE, 2004). A teoria da presença social indica que em qualquer interação entre duas partes, ambos os lados estão interessados em desempenhar um papel ou em algum tipo de interação social. A questão mais importante é, não apenas, 'estar ou não estar aqui', mas adquirir uma percepção sensorial da outra pessoa, uma consciência de extrema importância. A compreensão profunda deriva da percepção de estar junto, o que implica em uma relação intensa entre os participantes do ambiente mediado. Na teoria da presença social é assumido que em qualquer interação que envolve duas partes, ambos estão interessados e ambos desempenham determinadas funções como desenvolver e manter algum tipo de relação pessoal. A presença social é conceituada como o grau em que uma pessoa é percebida como 'autêntico' e 'estando lá', ou seja, a capacidade de uma pessoa tem de se projetar 
como 'autêntica' em um ambiente on-line e outros perceberam essa pessoa como se estivesse lá e de forma autêntica.

Nesse estudo, as categorias possuem a vantagem de permitir a análise direta das postagens, mostrando o fluxo comunicacional de informações e interações, além de permitir a busca das auto percepções dos usuários, como realizado em outros estudos baseados em questionários específicos.

\section{Desenho e construção do estudo}

Baseado em pesquisa exploratória, os seguintes passos foram concebidos para recolher respostas às nossas questões de pesquisa que trazem a evidência do uso de Facebook como uma ferramenta na escola e de um enfoque sistemático sobre o uso desta mídia social nos processos educativos.

Os passos básicos foram:

1. Seleção das Escolas: Por um lado, foi utilizado um exame de âmbito nacional brasileiro (chamado ENEM), a fim de selecionar as escolas mais bem classificadas em torno da nossa universidade. De fato, até três escolas foram escolhidas em cada cidade da região do $\mathrm{ABC}$ (que compreende sete cidades), embora algumas escolas tinham unidades em mais de uma cidade. A seleção filtrou 12 escolas. Na Finlândia, por outro lado, e escolha se baseou no exame nacional para matrículas concebido para classificar as melhores escolas. Desta forma, as escolas selecionadas são de diferentes partes da Finlândia, de escolas pequenas a grandes. Os resultados finais do exame são baseados nos resultados de testes obrigatórios sobre quatro assuntos fundamentais e realizados na primavera de 2015.

2. Perfil oficial no Facebook: Pesquisou-se o perfil oficial do Facebook conforme indicado no site de cada escola. Todas as escolas brasileiras selecionadas possuíam seu perfil. Uma das escolas finlandesas escolhidas não possuía perfil e foi substituída pela próxima da lista.

3. Coleta de Dados: As últimas 16 postagens de cada escola foram coletadas e inseridas em uma planilha do tipo EXCEL.

4. Definição das Categorias: As categorias listadas abaixo emergiram da leitura das postagens. Elas não foram definidas à priori com base em literatura, mas foram analisadas e definidas de acordo com sua pertinência aos objetivos do estudo. Após uma rodada inicial de leitura e análise, as categorias foram elencadas; após esse procedimento, elas foram refinadas e revisadas, e a listagem final configurada. Essa lista está descrita como segue:

a) Ação pedagógica: A postagem indica alguma ação feita por estudantes com objetivo de aprendizagem. 
Exemplo: "Os alunos prepararam delicioso pão como havia sido feito por um personagem de desenho animado. O espírito de partilha e de cooperação estava presente em todos os momentos".

b) Divulgação: Algo que a escola tem feito e o objetivo da mensagem foi para compartilhar as informações com sua comunidade.

Exemplo: "As secretárias foram homenageadas pela nossa equipe por causa do dia da secretária".

c) Evento: O objetivo do post era informar sobre um evento (já acontecido ou para acontecer). Porque os alunos ou professores estão envolvidos nele ou simplesmente para informar a comunidade quando o evento é importante.

Exemplo: "Próxima sexta-feira às 20h00, uma banda de rock se apresentará na nossa escola [...]".

d) Informativa: Para disseminar informações importantes.

Exemplo: "O tempo da refeição favorece encontros, conversas e transformações...".

e) Marketing: Informações postadas para estimular o público a fazer parte da escola, para alcançar novos alunos.

Exemplo: "O nosso exame de admissão para novos alunos acontecerá no próximo domingo, às 9 hs...". "Venha estudar em uma das melhores escolas em nossa região!"

f) Cumprimentos: o texto foi escrito para celebrar algumas pessoas especiais, estudantes, professores ou outras ocasiões significativas.

Exemplo: "Happy seniors citizen's day! October $1^{\text {st } " . ~ " F e l i z ~ D i a ~ d o s ~}$ Pais!"

5. Presença Social: Após esses passos, as postagens foram analisadas em termos do conceito de presença social, como discutido antes. Em resumo, três maiores categorias foram empregadas para classificar os posts. Elas foram:

a) Afetiva: uso de humor pelo autor da postagem, ou qualquer tipo de emoção, ou auto revelação, apresentando-se para os leitores;

b) Interativa: se o escritor tentou estimular o intercâmbio com o leitor, como comentar escrita anterior do leitor, ou fazer citações dele, questionamento de leitores, agradecer ou retribuir elogios;

c) Coesiva: aproximar-se dos leitores, usando vocativos, pronomes (como "nós" ou "nossos alunos" [...]), enviando saudações;

d) Neutra: quando as categorias anteriores não foram encontradas.

6. Contagem final: foi calculado o número total de postagens, o total de mensagens por escola, e por categoria.

Os dois pesquisadores deste estudo, em seus respectivos países, executaram as mesmas etapas metodológicas. Os dados foram coletados e inseridos em uma única planilha e foram elaboradas tabelas para permitir a análise comparativa. Uma questão importante foi compreender o significado exato das categorias escolhidas, ao serem aplicadas aos dados coletados. Como consequência, ambos investigadores poderiam utilizar as mesmas categorias e comparar os seus resultados. Além disso, um processo 
de rodada dupla foi feito, a fim de garantir que os conteúdo das mensagens foram precisamente alocados nas categorias, como proposto pelos pesquisadores de análise de conteúdo (KRIPPENDORFF, 2013).

As categorias listadas no item 'definição de categorias' se originaram na avaliação inicial do material. Este procedimento é baseado no método conhecido como grounded theory e postula que, após a leitura inicial, temas recorrentes são listados e agrupados em uma categoria mais ampla. Desta forma, se escolheu as categorias utilizadas neste estudo.

\section{Resultados e discussões}

Os resultados mostraram que as escolas usam o Facebook, principalmente para 'estar presente' nos meios de comunicação social, ao invés de pensar em estar 'socialmente presente', como discutido anteriormente. Por 'estar presente', queremos dizer que as escolas devem ter uma percepção da importância deste meio na sociedade dessa época e lutar para apresentar um 'rosto pessoal', pelo qual poderão ser reconhecidos. Este fato pode ser percebido pelo número de postagens na categoria 'neutro', como sendo a atividade predominante, independentemente do país.

Os dados brasileiros são apresentados nas Tabelas 3 e 4. A maioria das mensagens foi focada em falar sobre eventos $(29,9 \%)$, possivelmente para transmitir aos 'facebookers' a ideia de que a escola é uma organização ativa. Um tipo de divulgação que chamamos de 'ação pedagógica' - porque pode ter aplicações educacionais também foi medido, sendo notado entre os menos favorecidos com $13,6 \%$ de todas as mensagens.

Tabela 3 - Categorias encontradas nas escolas brasileiras

\begin{tabular}{|c|c|c|c|c|c|c|c|c|c|c|c|c|c|c|}
\hline CATEGORIAS / ESCOLAS & SC01 & $\mathrm{SCO} 2$ & $\mathrm{SCO3}$ & $\mathrm{SCO4}$ & SC05 & SC06 & $\mathrm{SCO}$ & $\mathrm{SC08}$ & Scog & SC10 & SC11 & SC12 & TOTAL & $\%$ \\
\hline Ação Pedagógica & 2 & 1 & 0 & 1 & 7 & 3 & 1 & 0 & 3 & 5 & 0 & 1 & 24 & 13.6 \\
\hline Divulgação & 1 & 1 & 0 & 1 & 1 & 0 & 2 & 4 & 12 & 6 & 6 & 6 & 40 & 22.6 \\
\hline Evento & 10 & 7 & 2 & 4 & 6 & 2 & 6 & 7 & 0 & 3 & 2 & 4 & 53 & 29.9 \\
\hline Informativa & 2 & 1 & 0 & 0 & 1 & 8 & 2 & 2 & 0 & 0 & 8 & 1 & 25 & 14.1 \\
\hline Marketing & 1 & 5 & 3 & 10 & 0 & 3 & 4 & 3 & 1 & 1 & 0 & 0 & 31 & 17.5 \\
\hline \multirow[t]{2}{*}{ Cumprimento } & 0 & 1 & 0 & 0 & 1 & 0 & 1 & 0 & 0 & 1 & 0 & 0 & 4 & 2.3 \\
\hline & & & & & & & & & & & & & 177 & \\
\hline
\end{tabular}

Fonte: Elaboração própria. 
No caso das escolas brasileiras, a presença social desempenhou um papel de coesão em $27 \%$ das mensagens. Esse resultado aponta para alguma compreensão da importância do relacionamento com a comunidade. No entanto, como mostra a Tabela 4 , até $62,7 \%$ de todas as postagens eram neutras, com uma média semanal de sete mensagens por escola e com duas escolas 'mais falantes' com 16 posts por semana.

Os dados das escolas finlandesas são apresentados nas tabelas 5 e 6 . Como se pode ver, o item 'eventos' foi o maior nas postagens, seguido pela categoria 'divulgação'. O número de postagens de 'eventos' varia muito de escola para escola. Houve uma escola em que todas as mensagens foram sobre eventos, ao passo que houve outra escola com apenas uma mensagem sobre pós-evento. A variação das mensagens na categoria ‘divulgação' foi mais equilibrada entre as escolas.

A categoria das 'ações pedagógicas' aparece em terceiro lugar, mostrando sua importância para as escolas da Finlândia, quando comparado com escolas brasileiras. No entanto, havia duas escolas que apresentaram mais de $50 \%$ das postagens como 'ação pedagógica'. Essa presença aumentou o percentual das mensagens na categoria de ação pedagógica, mas em outras escolas este número foi muito menor. As mensagens informativas ficaram em quarto lugar, mas houve três escolas, que não postaram mensagens de cunho informativo. O número de mensagens em categorias de 'marketing' e de 'cumprimento' foi baixo em todas as escolas.

Em termos de presença social, podemos observar que as escolas finlandesas tiveram maior número de mensagens totais, mas a 'mais falante' teve 11,2 mensagens por semana, número menor em comparação com a correspondente brasileira. Além disso, o número médio de mensagens por semana foi menor na Finlândia do que as escolas brasileiras, quase a metade em termos do total de mensagens. Uma das razões para o número menor nas postagens médias pode ser a diferença de penetração do Facebook entre os países estudados. Embora, a penetração da Internet na Finlândia é maior do que no Brasil, o Facebook é uma mídia social muito mais popular no Brasil do que na Finlândia. Assim, a utilização de outros meios de comunicação social pode ter afetado o número médio de mensagens. Também houve grandes diferenças entre as escolas finlandesas, quando comparamos suas postagens por semana. $\mathrm{O}$ maior número foi de 11,2 mensagens e os mais baixos 0,4 mensagens. 
Tabela 4 - Presença social de escolas brasileiras nas postagens do Facebook

\begin{tabular}{|r|c|c|c|c|c|c|c|r|r|r|}
\hline ESCOLA & AFETIVA & INTERATIVA & COESIVA & NEUTRA & TOTAL & INICIO & FIM & DIAS & SEMANAS & POST./SEM. \\
\hline SC01 & & & 3 & 13 & 16 & $18 / \mathrm{set}$ & $05 /$ out & 17 & 2.4 & 6.6 \\
\hline $\mathbf{S C 0 2}$ & 4 & 2 & 2 & 8 & 16 & $28 / \mathrm{set}$ & $05 /$ out & 7 & 1.0 & 16.0 \\
\hline $\mathbf{S C 0 3}$ & & & 4 & 1 & 5 & $02 / \mathrm{fev}$ & $05 /$ out & 245 & 35.0 & 0.1 \\
\hline $\mathbf{S C 0 4}$ & & 1 & 2 & 13 & 16 & $25 / \mathrm{ago}$ & $05 /$ out & 41 & 5.9 & 2.7 \\
\hline $\mathbf{S C 0 5}$ & 1 & & 9 & 6 & 16 & $28 / \mathrm{set}$ & $05 /$ out & 7 & 1.0 & 16.0 \\
\hline $\mathbf{S C 0 6}$ & & 6 & & 10 & 16 & $23 / \mathrm{set}$ & $05 /$ out & 12 & 1.7 & 9.3 \\
\hline $\mathbf{S C 0 7}$ & & 3 & 4 & 9 & 16 & $02 / j u$ & $05 /$ out & 95 & 13.6 & 1.2 \\
\hline $\mathbf{S C 0 8}$ & & & 5 & 11 & 16 & $04 / \mathrm{set}$ & $05 /$ out & 31 & 4.4 & 3.6 \\
\hline $\mathbf{S C 0 9}$ & & & 7 & 9 & 16 & $18 / \mathrm{set}$ & $05 /$ out & 17 & 2.4 & 6.6 \\
\hline $\mathbf{S C 1 0}$ & & & 5 & 11 & 16 & $21 / \mathrm{set}$ & $05 /$ out & 14 & 2.0 & 8.0 \\
\hline $\mathbf{S C 1 1}$ & & 1 & 3 & 12 & 16 & $22 / \mathrm{set}$ & $05 /$ out & 13 & 1.9 & 8.6 \\
\hline $\mathbf{S C 1 2}$ & & & 4 & 8 & 12 & $17 / \mathrm{set}$ & $05 /$ out & 18 & 2.6 & 4.7 \\
\hline & $\mathbf{5}$ & $\mathbf{1 3}$ & $\mathbf{4 8}$ & $\mathbf{1 1 1}$ & $\mathbf{1 7 7}$ & & & & & $\mathbf{7 . 0}$ \\
\hline
\end{tabular}

Fonte: Elaboração própria.

Tabela 5 - Categorias encontradas nas escolas finlandesas

\begin{tabular}{|l|r|r|r|r|r|r|r|r|r|r|r|r|r|r|}
\hline Categories & EE & ET & RE & HS & LU & HN & OL & GL & LY & KU & PU & KS & Total & $\%$ \\
\hline Pedagogical action & 2 & 3 & 1 & 11 & 0 & 7 & 0 & 3 & 1 & 4 & 1 & 1 & 34 & 17,7 \\
\hline Divulgation & 5 & 4 & 5 & 1 & 5 & 2 & 0 & 7 & 7 & 7 & 9 & 6 & 58 & 30,2 \\
\hline Event & 4 & 4 & 8 & 2 & 6 & 3 & 16 & 3 & 3 & 2 & 1 & 7 & 59 & 30,7 \\
\hline Informative & 4 & 3 & 2 & 2 & 5 & 0 & 0 & 1 & 0 & 1 & 3 & 1 & 22 & 11,5 \\
\hline Marketing & 0 & 2 & 0 & 0 & 0 & 3 & 0 & 0 & 3 & 1 & 2 & 0 & 11 & 5,7 \\
\hline Greeting & 1 & 0 & 0 & 0 & 0 & 1 & 0 & 2 & 2 & 1 & 0 & 1 & 8 & 4,2 \\
\hline & 16 & 16 & 16 & 16 & 16 & 16 & 16 & 16 & 16 & 16 & 16 & 16 & 192 & 100,0 \\
\hline
\end{tabular}

Fonte: Elaboração própria.

Parece que a maioria das escolas selecionadas na Finlândia não tem um objetivo claro para a sua utilização do Facebook. Elas possivelmente tinham criado uma conta pública no Facebook para 'estar presente', mas não usou o seu poder de conexão com as pessoas ou para criar melhor presença social. O público-alvo não foi claramente reconhecido: alunos, pais, ex-alunos, os novos alunos, professores ou outro grupo. Parece haver alguém responsável pela conta no Facebook que determina o que postar. $\mathrm{O}$ estilo das mensagens era geralmente neutro e não tinham como objetivo criar ligações estreitas entre a escola e os grupos de interesse. Apesar disso, em algumas escolas havia estudantes que comentassem de forma muito ativa no Facebook, embora as mensagens da escola não incentivassem a criação de interação. Assim, os alunos parecem ser nativos digitais em seu uso e participação nessa mídia, em comparação com membros 
da escola - ao menos os responsáveis pelas postagens - que são geralmente mencionados como imigrantes digitais.

A mídia social é mais do que um canal de comunicação. Ela é o elemento capaz de construir relacionamentos e deve ser entendida como possuindo essa característica mais importante. Um perfil oficial no Facebook pode ser usado para manter a comunidade escolar envolvida, participando da vida escolar, e ajudar as pessoas a cuidar e contribuir entre si. A importância da tecnologia não está em si mesma, porém se fundamenta em ajudar as pessoas a se tornarem melhores ou para fazer as coisas de forma melhor. Em termos de processo de aprendizagem, as escolas poderiam melhorar as ações pedagógicas, utilizando as mídias sociais a fim de obter o envolvimento da comunidade e experimentar possíveis estratégias para a aplicação desta tecnologia como uma ferramenta educacional.

A maior semelhança a se destacar, em termos de presença social, é a categoria 'chamada coesiva', que pode ser possivelmente interpretado como um desejo de se estimular a proximidade com a comunidade. Não se pode dizer que essa categoria coesiva é melhor do que as demais, mas cada categoria está associada a uma abordagem específica. Relacionamentos são construídos através do tempo e da confiabilidade, assim uma intimidade progressiva deve ser desenvolvida entre a escola e os seus grupos de interesse.

Tabela 6 - Presença social nas postagens das escolas finlandesas

\begin{tabular}{|r|r|r|r|r|r|r|r|r|r|r|}
\hline School & Affective & Interactive & Cohesive & Neutral & Total & Start & End & Days & Weeks & Posts/week \\
\hline EE & 2 & 0 & 6 & $\mathbf{8}$ & 16 & $01 / 10$ & $08 / 20$ & 225 & 32,1 & 0,5 \\
\hline ET & 0 & 0 & 2 & 14 & 16 & $09 / 28$ & $08 / 11$ & 45 & 6,4 & 2,5 \\
\hline RE & 1 & 0 & 9 & 6 & 16 & $08 / 28$ & $09 / 24$ & 28 & 4,0 & 4,0 \\
\hline HS & 0 & 1 & 2 & 13 & 16 & $11 / 05$ & $11 / 18$ & 14 & 2,0 & 8,0 \\
\hline LU & 0 & 0 & 1 & 15 & 16 & $08 / 26$ & $11 / 16$ & 52 & 7,4 & 2,2 \\
\hline HN & 1 & 1 & 0 & 14 & 16 & $08 / 11$ & $11 / 24$ & 75 & 10,7 & 1,5 \\
\hline OL & 2 & 1 & 0 & 13 & 16 & $11 / 15$ & $11 / 24$ & 10 & 1,4 & 11,2 \\
\hline GL & 3 & 1 & 6 & 6 & 16 & $08 / 28$ & $11 / 24$ & 89 & 12,7 & 1,3 \\
\hline LY & 1 & 1 & 3 & 11 & 16 & $01 / 30$ & $11 / 24$ & 297 & 42,4 & 0,4 \\
\hline KU & 0 & 0 & 8 & 8 & 16 & $10 / 24$ & $11 / 24$ & 31 & 4,4 & 3,6 \\
\hline PU & 1 & 1 & 6 & 8 & 16 & $10 / 22$ & $11 / 24$ & 34 & 4,9 & 3,3 \\
\hline KS & 1 & 0 & 3 & 12 & 16 & $09 / 21$ & $11 / 24$ & 65 & 9,3 & 1,7 \\
\hline & $\mathbf{1 2}$ & $\mathbf{6}$ & $\mathbf{4 6}$ & $\mathbf{1 2 8}$ & $\mathbf{1 9 2}$ & & & & & $\mathbf{3 , 4}$ \\
\hline
\end{tabular}

Fonte: Elaboração própria.

\section{Considerações finais}

O uso do Facebook pelos estudantes, professores e demais pessoas de interesse 
pelas escolas é um fato inegável da vida atual e seus líderes não podem ficar alheios a esses processos. Vários desafios têm sido indicados por pesquisadores da área sobre a introdução da tecnologia como ferramenta para a melhoria da aprendizagem, especialmente as mídias sociais, como Fewkes e McCabe (2012) que indicam o cuidado: "[...] the nature of multimedia can captivate students easily, but this visual engagement does not necessarily represent intellectual engagement." Contudo, as possibilidades do uso das mídias sociais como suporte educacional necessita aprofundamento teórico-prático e pode, certamente, se tornar um dos canais informacionais úteis para facilitar e motivar os processos de ensino e aprendizagem.

Por outro lado, a criação de perfis e postagens rotineiras pelas escolas pode indicar o esforço no sentido de se fazer presente, ainda que a efetiva presença social requeira mais do que simplesmente ter um perfil ou postar mensagens 'genéricas'. De fato, a realidade que realmente deve ser considerada é o estreitamente das relações com os 'amigos' virtuais da escola e, obviamente, com sua comunidade de aprendizes. Ainda, por relacionamento se compreende não apenas 'falar', mas principalmente aprender a ouvir, prestar atenção, compreender as mensagens nas entrelinhas, responder, interagir com cada um deles.

Sabe-se que a tecnologia tem o poder de potencializar as boas e más atitudes dos seres humanos. Desconsiderando-se aqueles que criam perfis falsos, as pessoas estão ansiosas para serem encontradas, ouvidas, estão prontas para relacionamentos sociais, porque somos 'seres sociais'. Atualmente, observam-se em todos os lugares as pessoas olhando e acionando, atentamente, seus pequenos e inteligentes dispositivos móveis, conectando-se com outros seres virtualmente presentes, provenientes de outras distâncias, trocando pensamentos e sentimentos, de forma fácil e rápida. Estar socialmente presente é muito mais do que se sentar à mesma mesa: demanda tomar parte em conversas, trocar ideias, conhecimentos, problemas, planos, se dispor a ajudar etc. Não é surpreendente que a geração dos nativos digitais - e demais agregados - se sente nas mesas virtuais e desfrutam das mesmas atividades mencionadas, intercambiando todo o tipo de reações em seus novos ambientes.

Esse estudo mostrou escolas em países diferentes com comportamentos similares e, provavelmente, buscando os mesmos resultados. Um aspecto importante deve ser ressaltado, que é o fato de se ter coletado os dados a partir do lado público nos perfis das escolas. Talvez outras possibilidades relacionais possam ser encontradas a partir da análise do lado privativo dos perfis, ou seja, nas postagens que ocorrem entre 
os amigos virtuais. Esse pode ser uma importante continuidade desse estudo.

A abordagem de se analisar os dados de mídia social das escolas, em termos de presença social, pode ser uma das contribuições desse trabalho, bem como os resultados obtidos.

\section{COMPARATIVE STUDY ON THE USE OF SOCIAL MEDIA IN SCHOOLS.}

Abstract: The presence of Facebook, an almost universal communication channel, is undoubtedly transforming schools. A study with twelve Brazilian and Finnish schools was conducted to understand how Facebook has been employed. The social presence theory was used as a theoretical analysis. The most important similarity found was the cohesive presence in both communities. Despite the data is coming from the public profiles of institutions, certainly other specific uses of social media by schools can be exploited.

Key words: Social media. Facebook. Social presence. Pedagogical action.

\section{REFERÊNCIAS}

BAREN, J. K. V.; IJSSELSTEIJN, W. Measuring presence: a guide to current measurement approaches. European Community under the Information Society Technologies Programme, 23 march 2004. Report for OmniPres project IST-200139237. Disponível em:

<http://www8.informatik.umu.se/ jwworth/PresenceMeasurement.pdf >. Acesso em: 17 jun. 2016.

BIOCCA, F. et al. Criteria and scope conditions for a theory and measure of social presence. In: ANNUAL INTERNATIONAL WORKSHOP ON PRESENCE, 4., 2001, Philadelphia. Proceedings... Philadelphia: Temple University, 2001. p.456-480.

CAVAZZA, F. Social media landscape 2014. Fred Cavazza Blog, 22 mayo 2014. Disponivel em: <http://www.fredcavazza.net/2014/05/22/social-media-landscape2014>. Acesso em: 17 jun. 2016.

COUROS, A.; HILDEBRANDT, K. Digital citizenship education in Saskatchewan schools. [S.1.]: Saskatchewan Ministry of Education, 2015.

FEWKES, A. M.; MCCABE, M. Facebook: learning tool or distraction? Journal of Digital Learning in Teacher Education, Londres, v. 28, n. 3, p. 1001-5901, Jul. 2012. GOULART, E. E. Mídias sociais: uma contribuição de análise. Porto Alegre: EDIPUCRS, 2014.

GOULART, E.; RAMOS, R.; CRUZ, L. O que falam de nós: um estudo de postagens no Twitter. Mediação, Porto Alegre, v. 14, n. 4, p.49-62, 2012. 
INTERNET WORLD STATISTICS. Internet use statistics. 2015. Disponivel em: <http://www.internetworldstats.com/stats2.htm>. Acesso em: 17 jun. 2016.

KIETZMANN, J. H. Unpacking the social media phenomenon: towards a research agenda. Journal of Public Affairs, [S.1.], v.12, n. 2, p.109-119, 2012.

KRIPPENDORFF, K. Content analysys: an introduction to its methodology. Thousand Oaks: SAGE, 2013.

LEE, K. M. Presence, explicated. Communication Theory, Hoboken, v. 14, n. 1, p.2750, fev. 2004.

LOMBARD, M.; JONES, M. T. Identifying the (Tele) presence literature. PsychNology Journal, Berlin, v. 5, n. 2, p.197-206, jan. 2007.

MILLENNIALS: confident: connected: open to change. Pew Research Center, Washington, 24 feb. 2010. Disponivel em:

<http://www.pewsocialtrends.org/2010/02/24/millennials-confident-connected-open-tochange/>. Acesso em: 17 jun. 2016.

PATRÍCIO, M. R.; GONÇALVES, V. Utilização educativa do Facebook no ensino superior. In: CONFERENCE LEARNING AND TEACHING IN HIGHER

EDUCATION, 1., 2010, Évora. Actas... Évora: Ed. da Universidade de Évora, 2010. Disponivel em: <https://bibliotecadigital.ipb.pt/bitstream/10198/2879/4/7104.pdf>. Acesso em: 17 jun. 2016.

ROSENTHAL, S. Social media institute: social media for schools. Langwitches, 27 september 2015. Disponivel em: <http://langwitches.org/blog/2015/09/27/social-mediainstitute-social-media-for-schools/>. Acesso em: 17 jun. 2016.

ROURKE, L. et al. Methodological issues in the content analysis of computer conference transcripts. International Journal of Artificial Intelligence in Education, Lansdale, v. 12, n. 1, p. 10-29, jan. 2001.

SCHÖN, S.; EBNER, M. Online-Lernangebote in den Lebensalltag integrieren. In: MERAL AKIN-HECKE, D. R. Lehrende arbeiten mit dem Netz. [S.1.]: Institut zur Förderung digitaler Mediennutzung, 2015. p. 282-285. 